\title{
Evaluation the Possibility of Using of miRNA-21 as a Prognostic and Predictive Factor in Colorectal Cancer (South Egypt Experience)
}

\author{
Ashraf Zedain', Hosny Badrawy², Mayada Fawzy¹, Dina Ismail Abd El Razik², Sanaa Saber¹, \\ Abeer Ibrahim ${ }^{*}$ (i)
}

${ }^{1}$ Department of Medical Oncology \& Hematological malignancy, South Egypt Cancer Institute, Assiut University, Assiut, Egypt ${ }^{2}$ Department of Clinical Pathology, South Egypt Cancer Institute, Assiut University, Assiut, Egypt

Email: *abelsayed40@gmail.com

How to cite this paper: Zedain, A., Badrawy, H., Fawzy, M., El Razik, D.I.A., Saber, S. and Ibrahim, A. (2020) Evaluation the Possibility of Using of miRNA-21 as a Prognostic and Predictive Factor in Colorectal Cancer (South Egypt Experience). Journal of Cancer Therapy, 11, 88-97.

https://doi.org/10.4236/jct.2020.112008

Received: December 26, 2019

Accepted: February 25, 2020

Published: February 28, 2020

Copyright $\odot 2020$ by author(s) and Scientific Research Publishing Inc. This work is licensed under the Creative Commons Attribution International License (CC BY 4.0).

http://creativecommons.org/licenses/by/4.0/

(c) (i) Open Access

\begin{abstract}
Background: Micro RNA 21 (miRNA-21) is overexpressed in numerous solid types of tumors, indicating that its biology is relevant to numerous types of cancer. However, in Colorectal Cancer (CRC) there is a lot of studies confirmed its use as diagnostic marker, other studies suggested it could be used as a predictive marker for $5 \mathrm{Fu}$ sensitivity so this study was conducted to verified the significance of using miRNA-21 as a prognostic and predictive factor in CRC. Patients and Methods: It is a prospective study that included 45 patients with stage I-IV CRC who attended at South Egypt Cancer Institute from April 2017 to October 2018. miRNA-21 was measured by (q PCR) from peripheral blood. Response to 5-FU containing regimens was assessed using RECIST criteria and its levels of expression were correlated to the clincopathological data, response and survival of the patients. Results: High expression of the miRNA21 was significantly associated with advanced stage of CRC $\mathrm{p}<0.001$, Lymph Node (LN) invasion $\mathrm{p}<0.001$, distant metastasis $\mathrm{p}<0.01$ lymphovascular (LVI) invasion $\mathrm{p}<0.01$ and pre-treatment level of Carcino Embryonic Antigen (CEA) $\mathrm{p}<0.031$. Also we found that high expression level significantly badly affected the response to $5-\mathrm{Fu}$ containing regimen $\mathrm{p}<$ 0.001 , DFS $p<0.01$ and OS $p<0.01$. Conclusion: miRNA-21 could be used as non-invasive prognostic and predictive marker in CRC.
\end{abstract}

\section{Keywords}

CRC, miRNA-21, Prognostic, Predictive

\section{Introduction}

Colorectal cancer remains the third most commonly diagnosed cancer in both 
men and women and represents one of the most frequent causes of cancer death [1] due to its diversity in the clinical course. And approximately $50 \%$ of patients with colorectal cancer die from distant metastases, and in particular liver metastases, within 10 years of the initial diagnosis [2].

Clinicians up to now still require recognizing prognostic markers with minimal invasion and with high sensitivity, to improve the survival rate of CRC patients [3] [4].

The advances of molecular biology have added to a better understanding of cancer development, predicting its evolution, therapeutic response and even developing molecular target [5]. Recent studies have proved that microRNA (miRNA) play important roles in tumor invasion and metastasis [3] [4] [5] [6] [7]. miRNA are small, non-coding RNA that block specific target genes in mammalian cells by suppressing translation [8]. Depending on the target gene, miRNA can act as either oncogenes or tumor suppressive genes [9]. Several studies results proved that the aberrant expression of specific miRNA can be used as potent prognostic and predictive markers in colorectal cancer [10] [11]. Because current prognostic markers of many cancers, including colorectal cancer, poorly predict metastatic progression or tumor response to chemotherapy, the identification of such prognostic markers is the subject of intense research.

Several miRNAs isolated from serum have been evaluated for their use as prognostic markers in CRC. A recent report revealed a panel of miRNAs (miR-21, let-7g, miR-31, miR-92a, miR-181b, and miR-203) that can be reliably used as prognostic marker for CRC, with $93 \%$ sensitivity and $91 \%$ specificity in comparison with the traditional markers (CEA and CA19-9) [12]. Furthermore, miR-21 has also been shown to be able to differentiate both adenoma and CRC from healthy controls [13] [14]. Also recent studies suggested that low miRNA-21 could sensitize CRC cells to 5-fu treatment [6] [15].

However these results are still under investigations and researches, the results are not mature enough to reach agreement or consensus about which miRNA would be used as prognostic or predictive marker.

As miRNAs are still under investigation in colorectal cancer, so we choose mi RNA21 to focus on it in our study as the previous studies' results about it were encouraging.

\section{Patients and Methods}

This is a prospective study was done on 45 CRC newly diagnosed who presented to South Egypt Cancer Institute in the period between May 2017 and October 2018 with median follow up period of 18 months. All patients included in this study will subject to: Full history taking and complete clinical examination, all patients staged according to the $8^{\text {th }}$ edition, 2017 revision of the TNM staging classification. All patients had complete laboratory investigation and CEA at diagnosis. Biopsy was taken whether by endoscopy or surgery and sent to the pathology laboratory as a routine and examined by expert pathologist.

Received 5FU based chemotherapy regimen either CAPOX, FOLFOX or FOLIRI 
according to their stage + the target therapy in the pantumomab in patients with metastatic colorectal with $\mathrm{K}$ ras wild type. Patients followed up by computed tomography scan (CT) on the chest and pelvi-abdomen every 3 months during the treatment and every 6 months during the follow up. The response interpreted according to the response evaluation criteria in solid tumors (RECIST) criteria. All patients have signed a written consent containing the detailed description of the study. The research program was approved by the ethical committee, South Egypt Cancer Institute, SCI-IRBIORG0006563N0 = 354 .

Before the start of the treatment we collected $2 \mathrm{ml}$ of peripheral blood sample via direct venous puncture with complete aseptic precautions and measured miRNA- 21 by q real time PCR.

\subsection{Methods of miRNA-21 Measurement}

miRNA was isolated from peripheral venous blood samples by using the miRN easy Mini kit from Qiagen, Hilden, Germany.

Isolated miRNA is converted to its complementary DNA (CDNA) by using TaqMan miRNA Reverse Transcription kit from Qiagen, Hilden, Germany.

Then relative quantification of miRNA expression is measured by performing Q-PCR amplification using Taqman Universal PCR Master MixII (2x) and Taqman small RNA Assay (20x) by 7500 fast real time PCR system, Thermo Fisher Scientific, USA.

\subsection{Analysis}

To calculate relative expression in RT-qPCR qPCR Ct values for:

1) The gene being tested: in control and experimental samples;

2) The housekeeping gene: in control and experimental samples.

- Take the average of the Cycle threshold (Ct) values for the housekeeping gene and the gene being tested in the experimental and control samples, returning 4 values. The 4 values are Gene being Tested Experimental (TE), Gene being Tested Control (TC), Housekeeping Gene Experimental (HE), and Housekeeping Gene Control (HC).

- Calculate $\Delta \mathrm{Ct}$ values for the experimental $(\Delta \mathrm{CTE})$ and control $(\Delta \mathrm{CTC})$ conditions. $\triangle \mathrm{CTE}$ is the differences between TE and HE (TE-HE) and $\triangle \mathrm{CTC}$ is the difference between TC and HC (TC-HC).

- Then, calculate $\Delta \Delta \mathrm{Ct}$ which is the difference between $\Delta \mathrm{CTE}$ and $\Delta \mathrm{CTC}$ $(\Delta \mathrm{CTE}-\Delta \mathrm{CTC})$ to arrive at the Double Delta Ct Value $(\Delta \Delta \mathrm{Ct})$

- Calculate fold change: Relative quantification (RQ) which is the value of $2^{\wedge}-\Delta \Delta \mathrm{Ct}$ to get the expression status. If $\mathrm{RQ}$ is more than 1 means expression of target is more than the control included in my experiment (up expressed) and if RQ is less than 1 means expression of target is less than the control (down expressed).

\section{Statistical Analysis}

Data was represented as numbers, percentages, means or median. All analyses 
were conducted using SPSS software version 21. Univariant factors were analyzed using the chi-square test for categorical variables, Cox's proportional hazard regression analyses were used to estimate hazard ratios (HRs) of death according to serum and tumor miRNA levels, unadjusted and adjusted for potential confounding factors for death, Assumptions of proportionality were confirmed for the Cox proportional hazards analyses by generating Kaplan-Meier survival curves (e.g., high- vs. low-expression groups) and by ensuring that the two curves did not intersect each other. All $p$ values are two-sided; $p$ less than or equal to 0.05 also we calculated the odd ratio (OR) between low and high expression groups.

\section{Results}

\subsection{The Clinico-Pathological Features of the Studied Patients}

We enrolled 45 patients with CRC newly diagnosed who presented to SECI out patients clinic from May 2017 to September 2018. Tumor characteristics of the study patients are shown in Table 1.16 patients (36\%) had high risk pathology

Table 1. Clinico-pathological character of the patients.

\begin{tabular}{|c|c|c|c|}
\hline \multicolumn{2}{|c|}{ Item } & $\mathbf{N}$ & (\%) \\
\hline \multicolumn{2}{|c|}{ Age (years), Mean \pm standard deviation } & 48.29 & \pm 15.86 \\
\hline \multirow{2}{*}{ Sex } & Male & 22 & $(49)$ \\
\hline & Female & 23 & $(51)$ \\
\hline \multirow{2}{*}{ Site of tumor } & Colon & 23 & $(51)$ \\
\hline & Rectum & 22 & $(49)$ \\
\hline Pathology & Low risk pathology & 29 & (64) \\
\hline \multirow{4}{*}{ Grade of tumor } & High risk pathology & 16 & $(36)$ \\
\hline & G1 & 7 & $(16)$ \\
\hline & G2 & 22 & $(49)$ \\
\hline & G3 & 16 & (36) \\
\hline \multirow{3}{*}{ Lymphovascular invasion } & Negative & 15 & $(33)$ \\
\hline & Positive & 19 & $(42)$ \\
\hline & Not assessed & 11 & $(24)$ \\
\hline \multirow{2}{*}{ Tissue invasion } & limited invasion & 7 & $(15)$ \\
\hline & Marked invasion & 38 & $(84)$ \\
\hline \multirow{2}{*}{ Lymph node invasion } & No & 13 & $(29)$ \\
\hline & $\mathrm{N}+$ & 32 & $(71)$ \\
\hline \multirow{2}{*}{ Distant metastasis } & M0 & 22 & $(49)$ \\
\hline & M1 & 23 & $(51)$ \\
\hline \multirow{2}{*}{ Stage } & Early stage & 12 & (27) \\
\hline & Advanced stage & 33 & $(34)$ \\
\hline \multirow{2}{*}{ CEA at presentation } & $<5 \mathrm{ng} / \mathrm{m}$ & 20 & (44) \\
\hline & $\geq 5 \mathrm{ng} / \mathrm{m}$ & 25 & (56) \\
\hline
\end{tabular}

Data was expressed in form of mean (SD) and frequency (percentage). 
(mucinous adenocarcinoma, signet ring carcinoma, mucoid adenocarcinoma with signet ring differentiation and poorly differentiated adenocarcinoma) and 29 patients (64\%) patients had low risk pathology (well differentiated adenocarcinoma and moderate differentiated adenocarcinoma) with 7 patients (16\%) had grade 1 (G1) tumor, 22 patients (49\%) had G2 tumor and 16 patients (35\%) had G3 tumor. 15 patients (33\%) had negative lymphovascular invasion (LVI), 19 patients (42\%) had positive LVI. 11 patients LVI not assessed.

Regarding the TNM staging, only 7 patients (16\%) had limited invasion (T1, T2) and 38 (84\%) patients had marked invasion (T3, T4). 13 (29\%) patients had N0 and $32(71 \%)$ had $\mathrm{N}+(\mathrm{N} 1, \mathrm{~N} 2, \mathrm{Nx}) .22(49 \%)$ patients presented with stage I-III, while 23 (51\%) presented with metastatic disease (Table 1). 25 (56\%) patients presented with high CEA level ( $\geq 5 \mathrm{ng} / \mathrm{ml}$ ) while 20 (44\%) patients with normal level $(<5 \mathrm{ng} / \mathrm{ml})$ (Table 1$)$.

\subsection{Expression of miRNA-21 and Its Correlation with Control and Clinico-Pathological Features}

Nineteen patients (42\%) had down expressed miRNA-21 level and 26 patients $(57.78 \%)$ had up expressed level with (cut off value $=1)$. We didn't find any significant association between the high expression of miRNA21 and age, sex, site of tumors, pathology type and tumors grads (Table 2). However, our results

Table 2. Corerelatioon between miRNA-21 expression and clicopathological data.

\begin{tabular}{|c|c|c|c|c|c|c|}
\hline \multirow{2}{*}{\multicolumn{2}{|c|}{ MiRNA21 }} & No & $\%$ & No & $\%$ & \multirow[b]{2}{*}{$\mathrm{p}$} \\
\hline & & \multicolumn{2}{|c|}{$\begin{array}{c}\text { Down expressed } \\
\mathrm{N}=19\end{array}$} & \multicolumn{2}{|c|}{$\begin{array}{c}\text { Up expressed } \\
N=26\end{array}$} & \\
\hline \multirow{2}{*}{ Pathology } & Low risk pathology & 12 & $(41)$ & 17 & $(59$ & \multirow{2}{*}{0.878} \\
\hline & High risk pathology & 7 & $(43)$ & 9 & $(56)$ & \\
\hline \multirow{3}{*}{$\begin{array}{l}\text { Differentiation } \\
\text { of tumor }\end{array}$} & Well differentiated & 4 & $(57)$ & 3 & $(43)$ & \multirow{3}{*}{0.609} \\
\hline & Moderate differentiated & 8 & $(36)$ & 14 & $(64)$ & \\
\hline & Poorly differentiated & 7 & $(44)$ & 9 & $(56)$ & \\
\hline \multirow{3}{*}{$\begin{array}{l}\text { Lymphovascular } \\
\text { invasion }\end{array}$} & Negative & 11 & $(73)$ & 4 & $(27)$ & \multirow{3}{*}{0.01} \\
\hline & Positive & 6 & $(32)$ & 13 & $(68)$ & \\
\hline & Not assessed & 2 & $(18)$ & 9 & $(82)$ & \\
\hline \multirow[b]{2}{*}{$\mathrm{T}$} & Limited invasion & 4 & $(57)$ & 3 & $(43)$ & \multirow[b]{2}{*}{0.433} \\
\hline & Marked invasion & 15 & (39) & 23 & $(61)$ & \\
\hline \multirow[b]{2}{*}{$\mathbf{N}$} & No & 11 & $(84)$ & 2 & $(15)$ & \multirow[b]{2}{*}{0.002} \\
\hline & $\mathrm{N}+$ & 8 & $(25)$ & 24 & $(75)$ & \\
\hline \multirow[b]{2}{*}{$\mathbf{M}$} & M0 & 13 & (59) & 9 & $(41)$ & \multirow[b]{2}{*}{0.01} \\
\hline & Metastatic & 6 & $(26)$ & 17 & $(74)$ & \\
\hline \multirow{2}{*}{ Stage } & Early stage & 10 & $(83)$ & 2 & $(17)$ & \multirow{2}{*}{0.001} \\
\hline & Advanced stage & 9 & $(27)$ & 24 & $(73)$ & \\
\hline
\end{tabular}

$\mathrm{T}=$ tumor invasion, $\mathrm{N}=$ lymph node invasion, $\mathrm{M}=$ distant metastasis. 
found significant association between high expression of miRNA21 and LVI $\mathrm{p}<$ 0.01 and the $\mathrm{LN}$ invasions p value $=0.002$ OR 16.5 (95\% CI: $2.99-90.86$ ). So patients with down expressed miRNA-21 were about 16 times more likely to have no lymph node invasion at time of diagnosis than those with up expressed miRNA21 (Table 2). Also we found statistical significant correlation with miRNA-21 and distant metastasis p value $=0.01$, OR 0.244 (95\% CI: $0.069-0.862$ ).

Patients with down expressed miRNA-21 are about four times less likely to being metastatic than those with up expressed miRNA-21 with (95\% CI: 1.16 14.49) (Table 2). The miRNA-21 was statistically significant stage $\mathrm{p}$ value $=$ 0.001, OR 13.33 (95\% CI: 2.44 - 73.02) (Table 2). There was also significant corelation between CEA and miRNA21 with p value $=0.01$, OR 3.86 (95\% CI: 1.11 - 13.46). Patients with down expressed miRNA21 were about three times more likely to have CEA at presentation $<5 \mathrm{ng} / \mathrm{ml}$ than those with up expression with (95\% CI: 1.11 - 13.46).

\subsection{The Correlation of the miRNA-21 with the Response to 5-Fu Based Chemotherapy}

The response to 5 -fu based regimen in metastatic patients showed statistically significant association with the low expression of miRNA21 levels $\mathrm{p}$ value $=0.001$.

\subsection{The Correlation of the miRNA-21 with the Survival}

Our results showed statistical significant difference in patients with low expression of miRNA-21 in patients with early disease (non metastatic) in their median DFS than those who presented with high expression $\mathrm{p}$ value $=0.024$ (Figure 1 ).

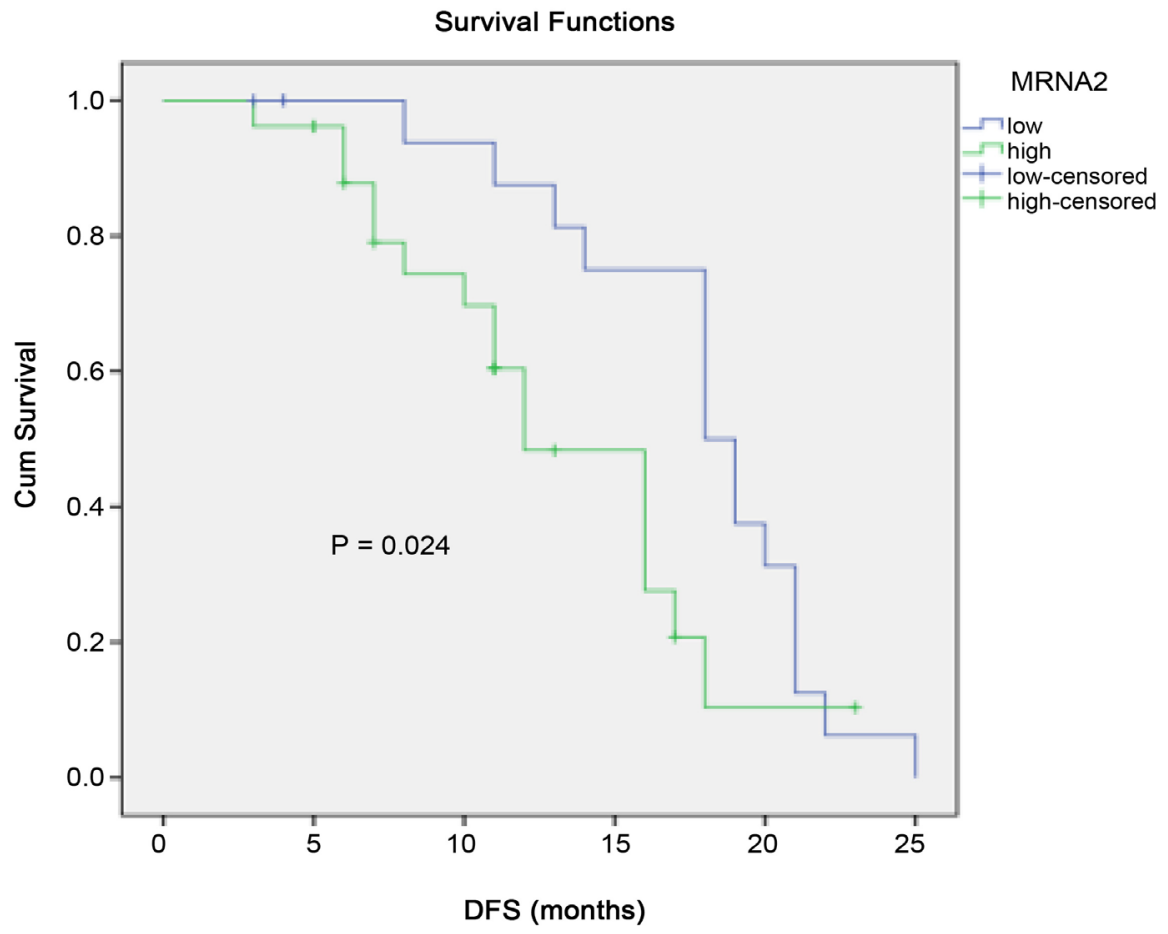

Figure 1. Correlation between the miRNA-21 and DFS. 
Hazard of relapse with up expressed miRNA-21 $=2.26$ (95\% CI: $1.05-4.85$ ). On the other hand, we didn't find significant difference between patients with low and high expression of miRNA-21 in advanced patients with metastases in their median PFS $\mathrm{p}$ value $=0.05$ Regarding the OS results showed significant difference between patients with low and high expression of miRNA 23 months (95\% CI: $21.1-25.53)$ vs. 20 months (95\% CI: 16.28 - 22.91), Hazard of death with up expressed miRNA21 $=4.22(95 \% \mathrm{CI}=0.901-19.65), \mathrm{p}$ value $=0.04$ (Figure 2$)$.

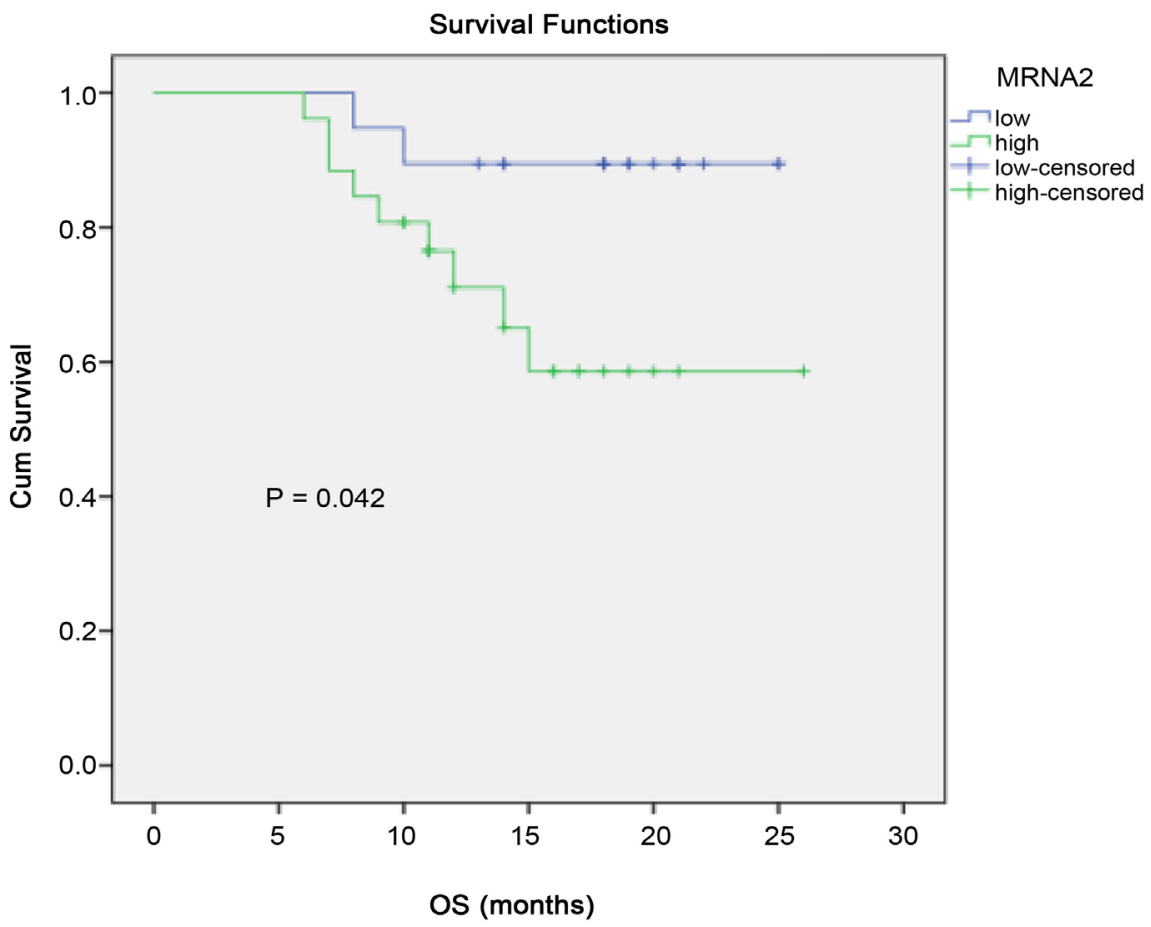

Figure 2. Correlation between the miRNA-21 and the OS.

\section{Discussion}

miRNA-21 is one of the most extensively investigated oncogenic miRNAs whose expression is frequently up-regulated in CRC [16]. It can down regulate several tumor suppressor genes, including PTEN [17].which acts as a lipid phosphatase to dephosphorylate phosphatidylinositol 3 trisphosphate (PIP3), antagonizing the PI3K/Akt pathway [6]. This pathway has an important effect on numerous biological functions, such as cell proliferation, adhesion, angiogenesis, migration, invasion, metabolism and anti-apoptosis [18]. This suggests the oncogenic role of miRNA-21 in CRC.

We conducted this study to investigate the miRNA21 as a prognostic and predictive factor in our cases of CRC.

There was no significant relationships in our study between the miRNA21 and the age, sex and site of tumor this was matched with previous studies by Zhang et al. and Bastaminejad et al. [19] [20].

Also we didn't find significant association between miRNA21 expression and pathological type and grade of differentiation this similar to the results of Mima 
et al. [21]. Our results might disagree with $\mathrm{Wu}$ et al. [22] who showed that miRNA-21 expression was significantly higher in patients with poor differentiation but this asymmetry probably explained by small sample size in our study.

There was no significant relationship in our study with the depth of invasion this was similar to results of Schee et al. [23] while Oue et al. [16] demonstrated that miRNA-21 expression was significantly increased in patients with T4, versus patients with $\mathrm{T} 1-\mathrm{T} 3$. These discrepancies might be explained by different sample size and may be different cut off values.

Patients with down expressed miRNA21 in our study are about four times less likely to being metastatic than those with high expression level. This was similar to results of multiple studies example of which Toiyama et al. [13] and Drusco et al. [24].

We found strong association between high expression of mi RNA and advanced stage and that was in line with previous results like Liu et al. [25] and Bastaminejad et al. [20] also significant association was found between the levels of expression of miRNA21 and LVI and this was explained by Fukushima et al. [26] who reported that miRNA inhibit programmed cell death protein 4 (PDCD4) which cause more invasion and intravasation [6].

Despite our results contradict previous meta-analysis [27] who reported no association between expression of mi RNA 21 and CEA levels. However, our results were in line with $\mathrm{Wu}$ et al. [22], as our data showed that patients with down expressed miRNA21 are about three times more likely to have CEA at presentation $<5 \mathrm{ng} / \mathrm{ml}$ than those with up expressed miRNA21, OR 3.86 (95\% CI= 1.11 13.46).

Regarding the response to 5FU based chemotherapy and survival, patients with high expressed level of miRNA21 were statistically significant insensitive to $5 \mathrm{FU}$ chemotherapy this was similar to results of Caramés et al. and Takaaki et al. [28] [29].

Moreover, we found strong significant association with DFS and OS which agree with previous researches like Tsukamotoet al. [30], Xia et al. [27] and Gao et al. [31]. But our results failed to find association with PFS however similar results were published before by Jie et al. [5].

\section{Conclusion}

miRNA-21 high expression is associated with bad risk factors and poor DFS and OS in CRC, also its high expression associated with decreased sensitivity to $5 \mathrm{Fu}$ based regimens. So miRNA-21 could be considered as an ideal non-invasive prognostic and predictive marker in CRC.

\section{Conflicts of Interest}

The authors declare no conflicts of interest regarding the publication of this paper.

\section{References}

[1] Yu, T., Guo, F., Yu, Y., et al. (2017) Fusobacterium nucleatum Promotes Chemore- 
sistance to Colorectal Cancer by Modulating Autophagy. Cell, 170, 548-563. https://doi.org/10.1016/j.cell.2017.07.008

[2] Holzel, D., Eckel, R. and Engel, J. (2009) Colorectal Cancer Metastasis Frequency, Prognosis, and Consequences. Der Chirurg, 80, 331-340.

https://doi.org/10.1007/s00104-008-1603-x

[3] Huang, Q., Gumireddy, K., Schrier, M., et al. (2008) The microRNAs miR-373 and miR-520c Promote Tumour Invasion and Metastasis. Nature Cell Biology, 10, 202-210. https://doi.org/10.1038/ncb1681

[4] Ma, L. and Weinberg, R.A. (2008) Micromanagers of Malignancy: Role of microRNAs in Regulating Metastasis. Trends in Genetics, 24, 448-456. https://doi.org/10.1016/j.tig.2008.06.004

[5] Jie, Y., Dongling, M., Andrew, F., et al. (2017) Expression Analysis of microRNA as Prognostic Biomarkers in Colorectal Cancer. Oncotarget, 8, 52403-52412. https://doi.org/10.18632/oncotarget.14175

[6] Yi, R., Li, Y., Wang, F.L., et al. (2016) MicroRNAs as Diagnostic and Prognostic Biomarkers in Colorectal Cancer. World Journal of Gastrointestinal Oncology, 8, 330-340. https://doi.org/10.4251/wjgo.v8.i4.330

[7] Wellner, U., Schubert, J., Burk, U.C., et al. (2009) The EMT-Activator ZEB1 Promotes Tumorigenicity by Repressing Stemness-Inhibiting microRNAs. Nature Cell Biology, 11, 1487-1495. https://doi.org/10.1038/ncb1998

[8] Bartel, D.P. (2004) MicroRNAs: Genomics, Biogenesis, Mechanism, and Function. Cell, 116, 281-297. https://doi.org/10.1016/S0092-8674(04)00045-5

[9] Zhang, W., Dahlberg, J.E. and Tam, W. (2007) MicroRNAs in Tumorigenesis: A Primer. The American Journal of Pathology, 171, 728-738. https://doi.org/10.2353/ajpath.2007.070070

[10] Schepeler, T., Reinert, J.T., Ostenfeld, M.S., et al. (2008) Diagnostic and Prognostic microRNAs in Stage II Colon Cancer. Cancer Research, 68, 6416-6424. https://doi.org/10.1158/0008-5472.CAN-07-6110

[11] Schetter, A.J., Leung, S.Y., Sohn, J.J., et al. (2008) MicroRNA Expression Profiles Associated with Prognosis and Therapeutic Outcome in Colon Adenocarcinoma. JAMA, 299, 425-436. https://doi.org/10.1001/jama.299.4.425

[12] Wang, J., Huang, S.K., Zhao, M., Yang, M., Zhong, J.L., Gu, Y.Y., Peng, H., Che, Y.Q. and Huang, C.Z. (2014) Identification of a Circulating microRNA Signature for Colorectal Cancer Detection. PLoS ONE, 9, e87451. https://doi.org/10.1371/journal.pone.0087451

[13] Toiyama, Y., Takahashi, M., Hur, K., Nagasaka, T., Tanaka, K., Inoue, Y., Kusunoki, M., Boland, C.R. and Goel, A. (2013) Serum miR-21 as a Diagnostic and Prognostic Biomarker in Colorectal Cancer. Journal of the National Cancer Institute, 105, 849-859. https://doi.org/10.1093/jnci/djt101

[14] Hollis, M., Nair, K., Vyas, A., Chaturvedi, L.S., Gambhir, S. and Vyas, D. (2015) MicroRNAs Potential Utility in Colon Cancer: Early Detection, Prognosis, and Chemosensitivity. World Journal of Gastroenterology, 21, 8284-8292. https://doi.org/10.3748/wjg.v21.i27.8284

[15] Yu, H.-M., Wang, C., Yuan, Z., et al. (2019) LncRNA NEAT1 Promotes the Tumorigenesis of Colorectal Cancer by Sponging miR-193a-3p. Cell Proliferation, 52, e12526. https://doi.org/10.1111/cpr.12526

[16] Oue, N., Anami, K., Schetter, A.J., et al. (2014) High miR-21 Expression from FFPE Tissues Is Associated with Poor Survival and Response to Adjuvant Chemotherapy in Colon Cancer. International Journal of Cancer, 134, 1926-1934. 
https://doi.org/10.1002/ijc.28522

[17] Leslie, N.R. and Downes, C.P. (2002) PTEN: The Down Side of PI 3-Kinase Signalling. Cellular Signalling, 14, 285-295. https://doi.org/10.1016/S0898-6568(01)00234-0

[18] Bader, A.G., Kang, S., Zhao, L., et al. (2005) Oncogenic PI3K Deregulates Transcription and Translation. Nature Reviews Cancer, 5, 921-929.

https://doi.org/10.1038/nrc1753

[19] Zhang, W., Zhang, T., Jin, R., et al. (2014) MicroRNA-301a Promotes Migration and Invasion by Targeting TGFBR2 in Human Colorectal Cancer. Journal of Experimental \& Clinical Cancer Research, 33, Article No. 113. https://doi.org/10.1186/s13046-014-0113-6

[20] Bastaminejad, S., Taherikalani, M., Ghanbari, R., et al. (2016) Investigation of microrna-21 Expression Levels in Serum and Stool as a Potential Non-Invasive Biomarker for Diagnosis of Colorectal Cancer. Iranian Biomedical Journal, 21, 106-113. https://doi.org/10.18869/acadpub.ibj.21.2.106

[21] Mima, K., Sukawa, Y., Nishihara, R., et al. (2016) Fusobacterium nucleatum and T-Cells in Colorectal Carcinoma. JAMA Oncology, 1, 653-661. https://doi.org/10.1001/jamaoncol.2015.1377

[22] Wu, Y., Song, Y., Xiong, Y., et al. (2017) MicroRNA-21 (Mir-21) Promotes Cell Growth and Invasion by Repressing Tumor Suppressor PTEN in Colorectal Cancer. Cellular Physiology and Biochemistry, 43, 945-958. https://doi.org/10.1159/000481648

[23] Schee, K., Boye, K., Abrahamsen, T., et al. (2012) Clinical Relevance of microRNA miR-21, miR-31, miR-92a, miR-101, miR-106a and miR-145 in Colorectal Cancer. BMC Cancer, 12, Article No. 505. https://doi.org/10.1186/1471-2407-12-505

[24] Drusco, A., Nuovo, G.J., Zanesi, N., et al. (2014) MicroRNA Profiles Discriminate among Colon Cancer Metastasis. PLoS ONE, 9, e96670. https://doi.org/10.1371/journal.pone.0096670

[25] Liu, G.H., Zhou, Z.G., Chen, R., et al. (2013) Serum miR-21 and miR-92a as Biomarkers in the Diagnosis and Prognosis of Colorectal Cancer. Tumor Biology, 34, 2175-2181. https://doi.org/10.1007/s13277-013-0753-8

[26] Fukushima, Y., Iinuma, H., Tsukamoto, M., et al. (2015) Clinical Significance of microRNA-21 as a Biomarker in Each Dukes' Stage of Colorectal Cancer. Oncology Reports, 33, 573-582. https://doi.org/10.3892/or.2014.3614

[27] Xia, X., Yang, B., Zhai, X., et al. (2013) Prognostic Role of microRNA-21 in Colorectal Cancer: A Meta-Analysis. PLoS ONE, 8, e80426. https://doi.org/10.1371/journal.pone.0080426

[28] Caramés, C., Cristóbal, I., Moreno, V., et al. (2015) MicroRNA-21 Predicts Response to Preoperative Chemoradiotherapy in Locally Advanced Rectal Cancer. International Journal of Colorectal Disease, 30, 899-906. https://doi.org/10.1007/s00384-015-2231-9

[29] Takaaki, M., Naoki, H., Yosuke, K., et al. (2017) MicroRNAs as Biomarkers in Colorectal Cancer. Cancers, 9, 124. https://doi.org/10.3390/cancers9090124

[30] Tsukamoto, M., Iinuma, H., Yagi, T., et al. (2017) Circulating Exosomal MicroRNA-21 as a Biomarker in Each Tumor Stage of Colorectal Cancer. Oncology, 92, 360-370. https://doi.org/10.1159/000463387

[31] Gao, S., Zhao, Z.-Y., Wu, R., et al. (2018) Prognostic Value of microRNAs in Colorectal Cancer: A Meta-Analysis. Cancer Management and Research, 10, 907-929. https://doi.org/10.2147/CMAR.S157493 